\title{
A New Method for Detecting the Time-Varying Nonlinear Damping in Nonlinear Oscillation Systems: Nonparametric Identification
}

\author{
T. S. Jang, ${ }^{1}$ Hyoungsu Baek, ${ }^{2}$ M. C. Kim, ${ }^{1}$ and B. Y. Moon ${ }^{3}$ \\ ${ }^{1}$ Naval Architecture and Ocean Engineering, Pusan National University, Busan 609-735, Republic of Korea \\ 2 Department of Mathematics, Massachusetts Institute of Technology, 183 Memorial Drive, Cambridge, \\ MA 02139, USA \\ ${ }^{3}$ Department of Naval Architecture, Kunsan National University, Gunsan 573-701, Republic of Korea
}

Correspondence should be addressed to T. S. Jang, taek@pusan.ac.kr

Received 11 July 2011; Accepted 6 September 2011

Academic Editor: Anuar Ishak

Copyright (C) 2011 T. S. Jang et al. This is an open access article distributed under the Creative Commons Attribution License, which permits unrestricted use, distribution, and reproduction in any medium, provided the original work is properly cited.

This paper presents an original method that can be used for identifying time-varying nonlinear damping characteristics of a nonlinear oscillation system. The method developed involves the nonparametric identification, in which only the system responses, namely, displacement and velocity need to be known for the identification. However, the method is concerned with a Volterratype integral equation of the first kind, which leads to an instability of numerical solutions. That is, the solutions identified lack stability properties. In order to overcome the difficulty, a stabilization technique is applied to the identification process. A numerical example comprising a highly nonlinear system is examined to demonstrate the workability of the proposed method for the time-varying damping identification.

\section{Introduction}

As a mathematical modeling, the identification of system damping has received increased attention in the fields of science and engineering over the last few decades. A proper modeling of nonlinear damping characteristics is important in many practical applications. For example, it is essential for the response prediction, real-time monitoring of structure, load identification, and so forth, in regard to nonlinear physical systems.

Many identification methods have been investigated for identifying dampings. Iourtchenko et al. [1] successfully proposed an identification method for the case of parametric excitation. By using a measured stationary response, a procedure for the inservice identification of the damping characteristics was suggested on the basis of the 
stochastic averaging method of Iourtchenko and Dimentberg [2]. There are challenging issues associated with the modeling and identification of nonlinear damping systems [3-6].

Even if significant progress has been made, many of challenging problems concerning the damping identification still await their solutions. Most of (nonlinear) damping identification is applied to autonomous systems: that is, the dampings we shall identify do not explicitly involve time. Recently, however, much attention has been paid to the time-varying damping identification of nonlinear dynamic models in various fields of engineering. For example, the estimation of time-varying damping is crucial in assessing the risk of negative aeroelastic damping in a wind turbine [7]. In this paper, we are concerned with the identification of such time-varying damping.

We propose an original method for identifying time-varying nonlinear damping in nonlinear dynamical systems. The method proposed here is a novel one with remarkable improvements. First, based on the fact that the method is of nonparametric identification, any "priori" assumption of the functional form of time-varying damping is not required. Measured system responses of displacement and velocity are sufficient for the identification process of the method. Second, the method converts a measured displacement into a pseudodisplacement whose relationship with the time-varying damping is linear. Third, a numerical procedure of the method utilizes a stabilization technique in order to solve a problem of numerical instability occurred in the identification process. The stabilization technique is known as regularization, the idea of which is briefly to replace integral equations of the first kind by nearby second kind integral equations. Fourth, because the method does not depend upon small parameters at all, it overcomes the limitations and disadvantages of the technique of perturbation expansion. Finally, we demonstrate the workability of the method through a numerical experiment.

\section{The Equation of Motion}

A nonlinear oscillator with a time-varying nonlinear damping is considered, in which the governing equation is described as a second-order nonlinear differential equation:

$$
m \ddot{y}+k y=\lambda(t) f(\dot{y})+r(y)
$$

where the symbols of $m$ and $k$ indicate constants. With $\alpha$ and $\beta$ real values, an initial condition is imposed as

$$
y(0)=\alpha, \quad \dot{y}(0)=\beta .
$$

Equation (2.1) together with (2.2) constitutes an initial value problem for the motion of the oscillator. In this paper, we assume that the time-varying nonlinear damping of systems is characterized by a separable function, $\lambda(t) f(\dot{y})$, as in $(2.1)$, where $f(\dot{y})$ denotes a general nonlinear function of the velocity $\dot{y}$ and $\lambda(t)$ its time-varying coefficient.

We will transform the differential representation of (2.1) into an integral one, because sometimes mathematically an appropriate manipulation of an integral expression has advantages over that of differential one [8-14]. For example, integral operators appearing in integral expressions are in general stable, whereas differential operators of differential equations are usually unstable. Using a Green's function method as well as a method of 
variation of parameters [15], we are able to find an integral relationship, equivalent to (2.1), as follows $[9,10]$ :

$$
y(t)=\alpha y_{1}(t)+\beta y_{2}(t)+\int_{0}^{t} K(t, \tau)[\lambda(t) f(\dot{y})+r(y)] d \tau
$$

With Wronskian $W=y_{1} \dot{y}_{2}-\dot{y}_{1} y_{2}$, the kernel $K$ in (2.3) is expressed as

$$
K(t, \tau) \equiv \frac{\left[y_{1}(\tau) y_{2}(t)-y_{1}(t) y_{2}(\tau)\right]}{m W(\tau)}
$$

in which $y_{1}$ and $y_{2}$ are functions that satisfy the following equations:

$$
\begin{array}{ll}
m \ddot{y}_{1}+k y_{1}=0, & y_{1}(0)=1, \quad \dot{y}_{1}(0)=0, \\
m \ddot{y}_{2}+k y_{2}=0, & y_{2}(0)=0, \quad \dot{y}_{2}(0)=1 .
\end{array}
$$

\section{Integral Equation}

Equation (2.3) is rewritten as

$$
y(t)-\alpha y_{1}(t)-\beta y_{2}(t)-\int_{0}^{t} K(t, \tau)[r(y(\tau))] d \tau=\int_{0}^{t} \tilde{K}(t, \tau) \lambda(\tau) d \tau
$$

where a new kernel $\tilde{K}$ is defined as

$$
\widetilde{K}(t, \tau) \equiv K(t, \tau) f[\dot{y}(\tau)]
$$

Since all the quantities on the left-hand side of (3.1) are known, (3.1) is an integral equation for $\lambda(t)$. Let us call the left-hand side of (3.1) a pseudodisplacement $\eta$ in this study, that is,

$$
\eta(t) \equiv y(t)-\alpha y_{1}(t)-\beta y_{2}(t)-\int_{0}^{t} K(t, \tau)[r(y(\tau))] d \tau .
$$

It then immediately follows that

$$
\eta(t)=\int_{0}^{t} \tilde{K}(t, \tau) \lambda(\tau) d \tau
$$

from (3.1) and (3.3).

The physical meaning of the integral equation (3.4) is that if we know the system's responses of the displacement $y$ and its velocity $\dot{y}$ in advance, we can recover (or identify) the time-varying damping coefficient $\lambda(t)$ in (2.1) by solving (3.4). In practice, conventional 
measurement devices enable the measuring of system's responses to be performed in an accurate manner.

Even though the oscillators with time-varying damping, considered herein, are "nonlinear" as exhibited in (2.1), the present problem to identify the oscillators' time-varying damping turns out to be "linear": that is, we establish a linear (integral) relationship between the pseudodisplacement $\eta$ and the unknown $\lambda(t)$ through (3.4).

\section{Uniqueness}

Prior to the discussion of a solution procedure for (3.4), physically it plays an important role to verify that the time-varying coefficient $\lambda(t)$ in (3.4) can be identified (or determined) in a really unique manner. For the proof of uniqueness, we first remind that the integral equation of (3.4) is linear in $\lambda(t)$. It thus suffices to show that the vanishing of the left-hand side of (3.4) (i.e., $\eta \equiv 0$ ) implies $\lambda \equiv 0$ in (3.4).

Let us assume that $\eta$ in (3.3) vanishes. That is,

$$
\eta(t)=y(t)-\alpha y_{1}(t)-\beta y_{2}(t)-\int_{0}^{t} K(t, \tau)[r(y(\tau))] d \tau=0,
$$

or

$$
y(t)=\alpha y_{1}(t)+\beta y_{2}(t)+\int_{0}^{t} K(t, \tau)[r(y(\tau))] d \tau
$$

Equation (4.2) is then a nonlinear integral equation that $y$ should satisfy. Recalling that (2.1) is equivalent to (2.3), the differential equation which is equivalent to (4.2) is

$$
m \ddot{y}+k y=r(y) .
$$

Comparing (2.1) and (4.3), we instantly arrive at

$$
\lambda \equiv 0
$$

This completes the proof. Thus, it has been shown that (3.4) has a unique solution. This means that we can recover the time-varying damping coefficient $\lambda(t)$ in $(2.1)$ in a unique way, once we know the measured responses of the displacement $y$ and velocity $\dot{y}$.

\section{Lack of Stability}

Although we have found that the present (inverse) problem of determining $\lambda(t)$ is one-toone, there still remains the question of the stability of the solution. That is, it is necessary to check whether the solution depends on the measured response data (displacement $y$ and velocity $\dot{y}$ ) in a continuous manner.

According to the theory of first-kind integral equations, the "first" kind Volterra integral equation with a regular kernel such as $\widetilde{K}(t, \tau)$ in (3.2) is ill-posed in the sense of 
stability [16-18]. Thus, the solution to (3.4) lacks stability properties. This means that the presence of noise in the measurement of $y$ and $\dot{y}$ can be greatly amplified in the numerical solution of $\lambda(t)$, creating unreliable solutions.

This (undesirable) phenomenon is in sharp contrast to the case of the usual wellposed integral equations of the "second" kind: conventional numerical methods are suitable for solving the usual well-posed equations, but they cannot be used to solve the ill-posed problem of (3.4) because of the lack of solution stability [16-18]. In fact, the direct (numerical) discretization of ill-posed systems such as (3.4) would result in ill-conditioned numerical systems with an extremely large condition number [8, 18-22].

\section{Computing $\lambda(t)$}

As mentioned in the previous section, even though many numerical methods have been developed for solving integral (or differential) equations, they are usually limited to wellposed (stable) problems. Thus, in order to solve the problems which lack stability properties, a special numerical procedure, suitable for the unstable problems, is required. To this end, we suggest the use of a stabilization technique, known as regularization. In this study, we use Tikhonov regularization to suppress the solution instability caused by the lack of stability properties.

\subsection{Tikhonov Regularization}

We briefly explain a procedure for Tikhonov regularization, the results from which will be presented at later section. According to Tikhonov [16], we construct a functional having an artificial dissipative function $\Omega$ for the solution for the integral equation of (3.4) for $T>0$,

$$
F(\lambda)=\left\{\int_{0}^{T}\left|\int_{0}^{t} \tilde{K}(t, \tau) \lambda(\tau) d \tau-\eta(t)\right|^{2} d t\right\}^{1 / 2}+\mu \cdot \Omega(\lambda)
$$

where the constant $\mu$ is called the regularization parameter, which is a positive real number. In this study, the functional form of $\Omega$ in (6.1) is chosen as follows:

$$
\Omega(\lambda)=\int_{0}^{T}\left|\frac{d^{2} \lambda}{d t^{2}}\right|^{2} d t
$$

We are able to minimize the (Tikhonov) functional $F$ in (6.1) by using the orthogonal structure of an infinite dimensional Hilbert space. This leads to

$$
\mu \frac{d^{4} \lambda}{d t^{4}}+L^{*} L(\lambda)=L^{*}(\eta)
$$


Table 1: Three test cases.

\begin{tabular}{lc}
\hline Case & $\lambda(t)$ \\
\hline 1 & $0.1\left(1+e^{-0.25(t-7)^{2}}\right)$ \\
2 & $0.1(1+0.5 \sin (t))$ \\
3 & $0.1(1+0.5 \sin (2 \pi t)) e^{-0.1(t-10)^{2}}$ \\
\hline
\end{tabular}

$L$ in (6.4) denotes an integral operator, whose kernel is $\widetilde{K}(t, \tau)$ in (3.2): that is,

$$
L(\xi)=\int_{0}^{t} \tilde{K}(t, \tau) \xi(\tau) d \tau,
$$

for an arbitrary function $\xi(t)$. The symbolic notations for $L^{*}$ in (6.3) is the adjoint operator of the $L[8,9]$. Due to the role of the artificial function $\mu \cdot \Omega$ introduced in (6.1), we can arrive at the integrodifferential equation of (6.3), whereas the original equation remains the "first" kind integral equation of (3.4). This means that the solution for (6.3) is stable, because the integrodifferential equation (6.3) is known to be well-posed.

\section{Numerical Experiment}

\subsection{A Model Equation}

In this section, we demonstrate the workability of the method proposed for the indirect measurement of time-varying damping. We begin with a model differential equation for the time-varying system of (2.1):

$$
\ddot{y}+\lambda(t)|\dot{y}| \dot{y}+y+0.1 y^{3}=0 .
$$

Recalling (3.2), we note that (7.1) is involved in the nonlinear function $f$ in (3.2) which has the following form:

$$
f[\dot{y}(\tau)]=|\dot{y}(\tau)| \dot{y}(\tau)
$$

Equation (7.2) is a typical functional form of the quadratic nonlinear damping arising in nonlinear dynamics of oscillations. Here, we list three cases of different $\lambda(t)$ 's for the present numerical experiment as shown in Table 1 . In case 1, a time-varying damping coefficient is set to $\lambda(t)=0.1\left(1+\mathrm{e}^{-0.25(t-7)^{2}}\right)$, which is bell-shaped centered at $t=7$. In contrast to such short transient period of the damping coefficient, case 2 has a periodic damping coefficient of $\lambda(t)=0.1(1+0.5 \sin (t))$. Case 3 has the combined feature of the cases 1 and 2 , that is, a periodic oscillation within a slowly varying smooth envelope. The damping coefficients $\lambda(t)$ of all the three cases are plotted in Figure 1. 


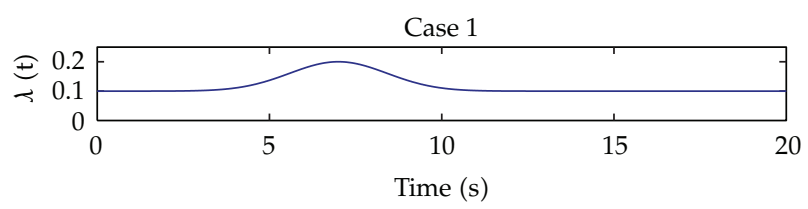

(a)

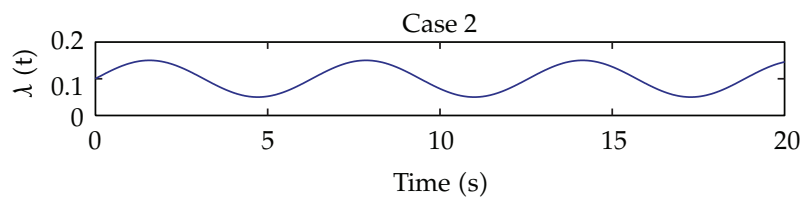

(b)

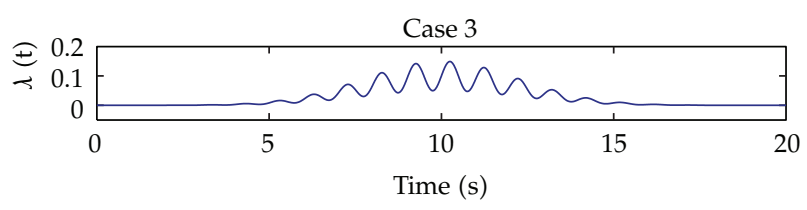

(c)

Figure 1: Time-varying damping coefficients of three test cases.

\subsection{System Responses}

In order to solve the (inverse) problem in (3.4), measurements of displacement and velocity are needed as explained in Section 3. In general, such data is obtained by numerical solutions of the (direct) problem in (7.1). The system responses of the numerical solutions can be found by using various usual numerical integration schemes of nonlinear ordinary differential equations.

In this study, the solution or measurement was obtained by using a second-order Runge-Kutta integration scheme, where the initial conditions are imposed as

$$
y(0)=0, \quad \dot{y}(0)=1 .
$$

Small time steps and high-order Runge-Kutta integration methods are tested and we confirmed that the measurement, that is, numerical solution of the equation, is accurate in this study. In practice, the measurement of motion responses is always degraded to a certain extent by noise. Thus, it may be impossible to determine the left-hand side of (3.4) exactly. However, in this numerical experiment, we assume that the noise is of the order of discretization errors.

As an example of a typical measurement, system responses are shown in Figures 2 and 3. Due to positive system damping, the phase diagram shows a gradual decay of system energy (spiral-in in the phase plot). The final time $T$ is set to 20 seconds. Although it has nothing to do with an inverse problem itself, numerically a large final time $T$ with small time step will result in a large discretization numerical operator, say, a large matrix 


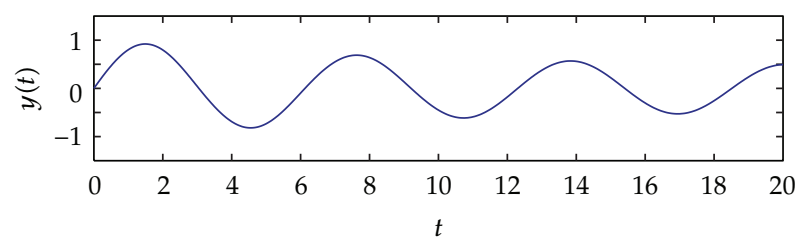

(a)

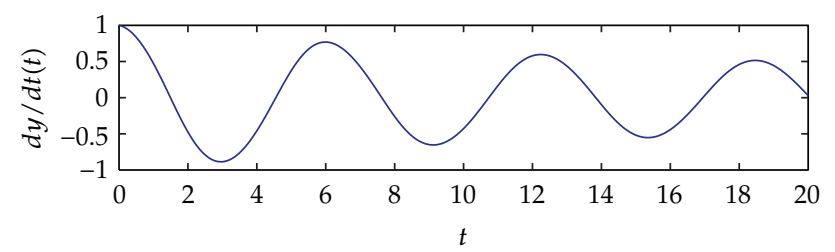

(b)

Figure 2: Time history of displacement and velocity (case 1).

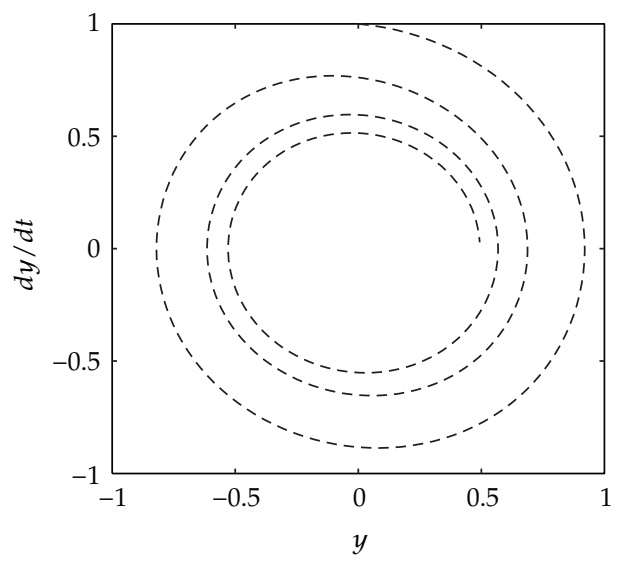

(a)

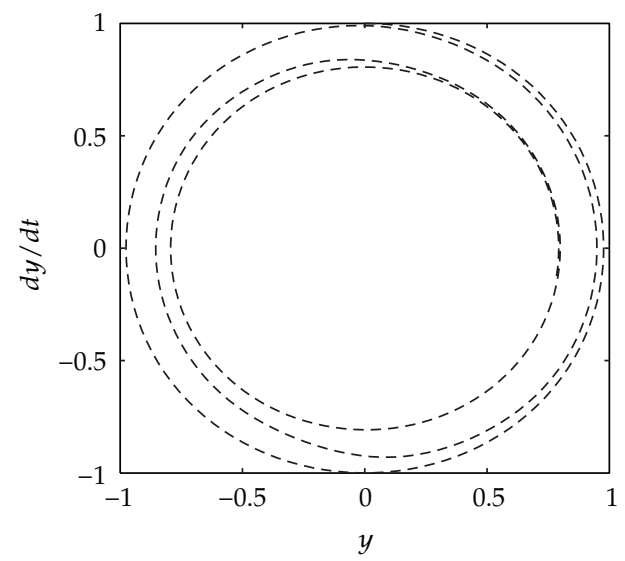

(b)

Figure 3: Phase diagrams (case 1 in (a) and case 3 in (b)).

by a discretization of $L$ in (6.3). In such situations, an iterative method like the Landweber regularization [9] can be very time-consuming and noniterative methods are preferred. This is a reason we present Tikhonov regularization in Section 6, which is a noniterative method.

\subsection{Recovering $\lambda$}

$\lambda(t)$ for three different regularization parameters of $\mu$ are illustrated in Figure 4 in which the Tikhonov regularization of (6.3) is used for the recovering. For the cases 2 and 3 , the recovered $\lambda(t)$ are depicted in Figures 5 and 6, respectively. Figures 4-6 show that the recovered results are in good agreement with the exact ones. But there is a small difference near the end points, 


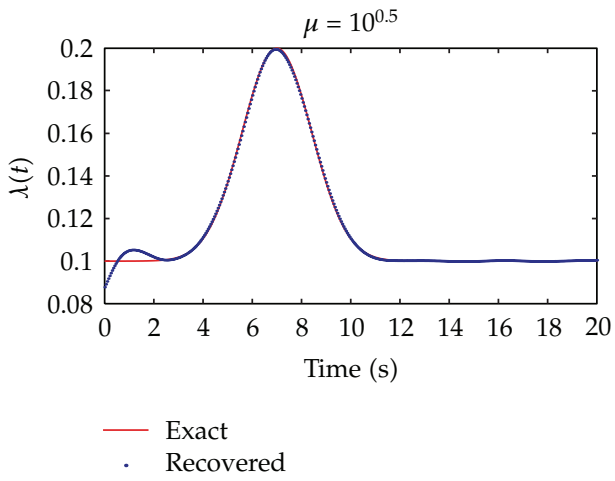

(a)

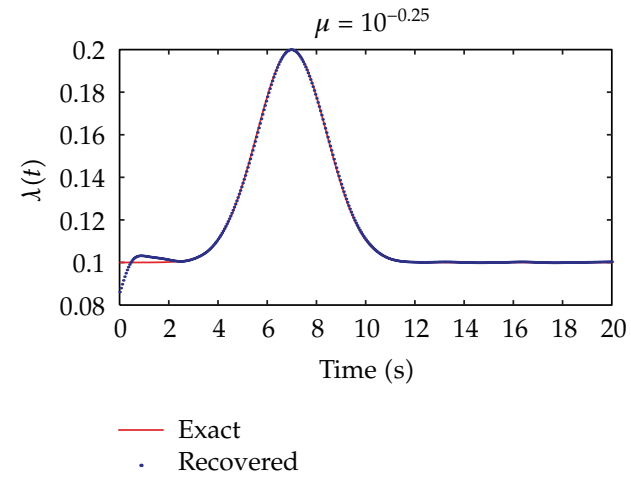

(b)

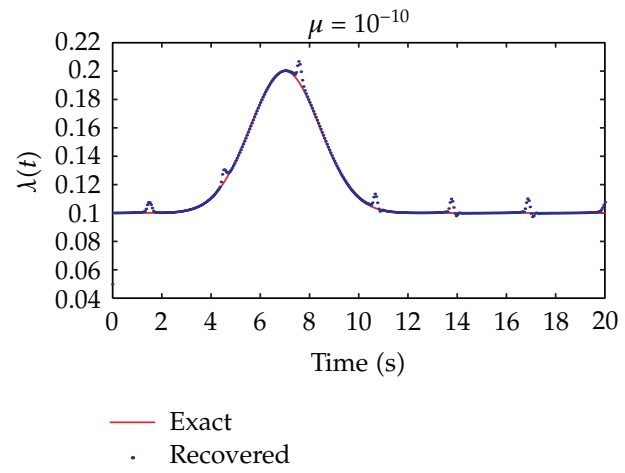

(c)

Figure 4: Recovered damping coefficient $\lambda(t)$ of case 1 (from (a) to (c) the regularization parameter).

which may be caused by nearly zero main diagonals involving a numerical discretization matrix for $L$ in (6.3). When $\mu$ is too large, the smoothness is enforced so strongly that the recovered $\lambda(t)$ cannot follow the sinusoidal (rapid) changes. When $\mu$ is too small, however, (3.4) is of a more dominating factor of minimization; hence, $\lambda(t)$ follows the details of the damping coefficient. However, erroneous peaks appear at times when $\dot{y}(t)$ is nearly zero due to the ill-posedness.

In this section, we demonstrate that the time varying damping can be covered when the regularization parameter $\mu$ is adjusted properly; in all cases, the time-varying coefficients are recovered successfully whether $\lambda(t)$ has either short-period changes, periodic changes, or both (periodic changes with amplitude modulation).

\section{Discussion}

We found that the formalism here has quite different characters from other related studies [8-14]; in the present study the kernel $\widetilde{K}(t, \tau)$ in (3.4) depends on the data. More specifically, $\widetilde{K}(t, \tau)$ in (3.2) includes system response data through $f[\dot{y}(\tau)]=|\dot{y}(\tau)| \dot{y}(\tau)$; this may look naive and harmless. However, the velocity $\dot{y}$ in (3.2) may be close to zero, which may induce the unwelcome small difference near the end points in Figures 4-6, just mentioned 


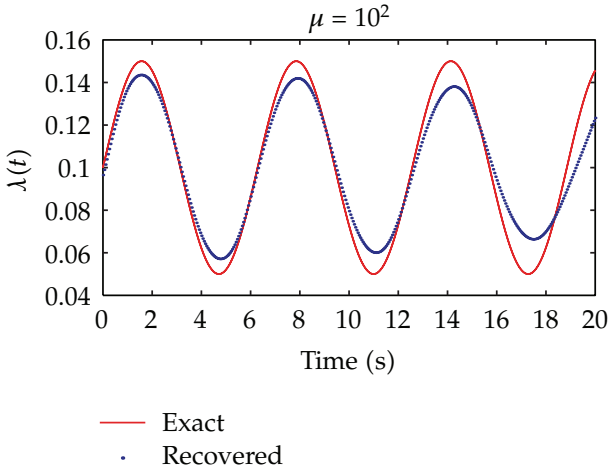

(a)

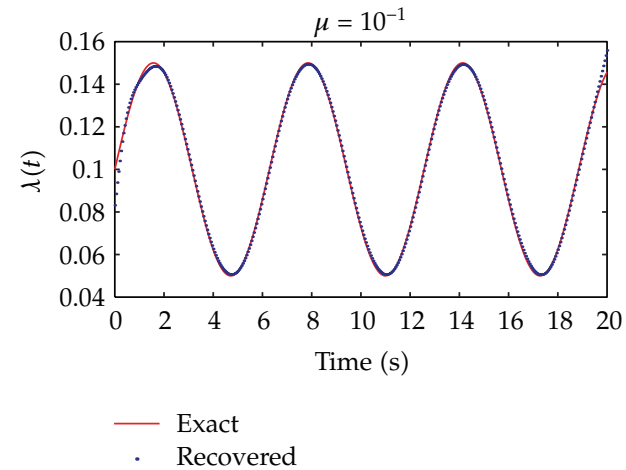

(b)

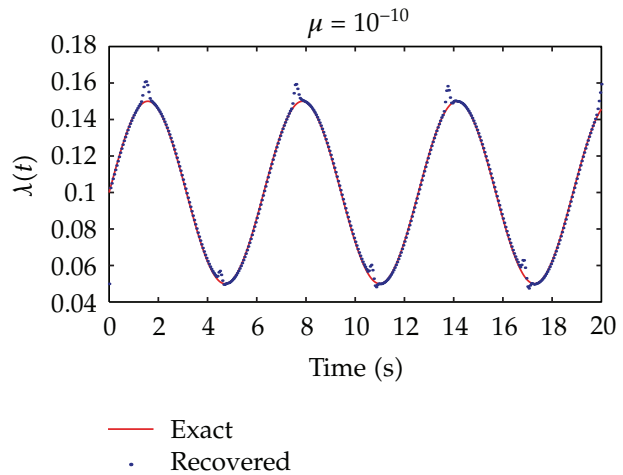

(c)

Figure 5: Recovered damping coefficient $\lambda(t)$ of case 2 (from (a) to (c) the regularization parameter).

above. Moreover, this velocity data is contaminated with a noise. Such data dependency of kernel causes an additional difficulty in the regularization, giving rise to sharp peaks in the recovered $\lambda(t)$.

\section{Conclusion}

The basic idea underlying the present study can be summarized as a fundamental question. Is it mathematically possible to identify (or recover) the time-varying damping characteristic of a nonlinear oscillation system, if we obtain "two" sets of motion response data of measured displacement and velocity? As a first step, we have answered the above question by assuming that the time-varying nonlinear damping of the system is separable: that is, it is expressed as a multiplication of a time-varying coefficient $\lambda(t)$ by a nonlinear function $f(\dot{y})$. Based on the separability assumption, we present a general method for identifying the timevarying coefficient $\lambda(t)$ of the system. Although a highly nonlinear system is provided as an example, the proposed method achieves a reasonably good approximation for $\mathcal{\lambda}$ in a stable manner. 


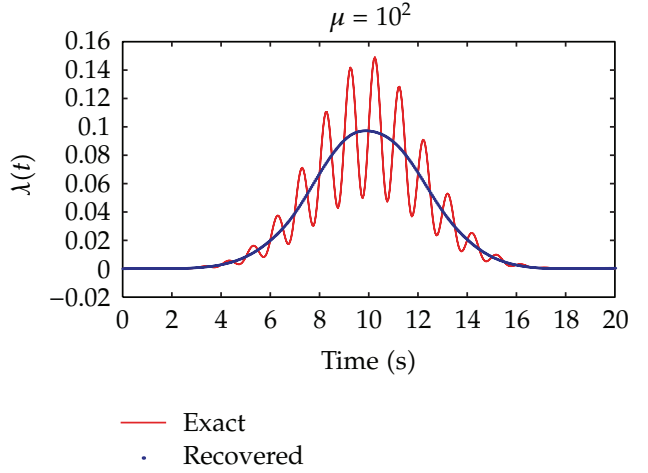

(a)

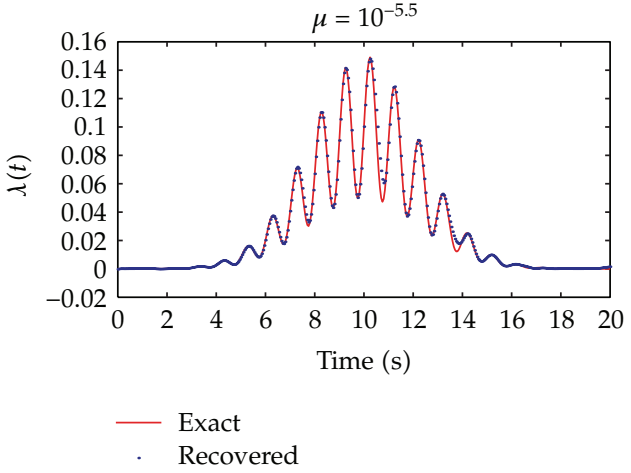

(b)

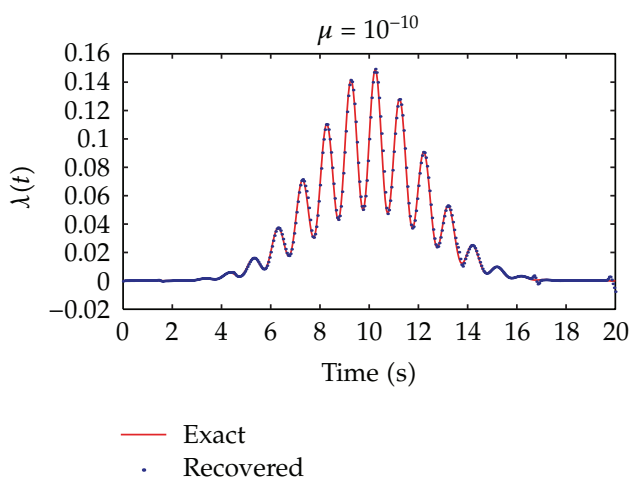

(c)

Figure 6: Recovered damping coefficient $\lambda(t)$ of case 3 (from (a) to (c) the regularization parameter).

\section{Acknowledgments}

This research was supported by Basic Science Research Program through the National Research Foundation of Korea (NRF) funded by the Ministry of Education, Science and Technology (Grant no.: 2011-0010090). And this research was supported by Leading Foreign Research Institute Recruitment Program through the National Research Foundation of Korea (NRF) funded by the Ministry of Education, Science and Technology (MEST) (Grant no.: K20901000005-09E0100-00510).

\section{References}

[1] D. V. Iourtchenko, L. Duval, and M. F. Dimentberg, "The damping identification for certain SDOF systems," in Proceedings of the SECTAM-XX, Developments in Theoretical and Applied Mechanics, Auburn University, 2000.

[2] D. V. Iourtchenko and M. F. Dimentberg, "In-service identification of non-linear damping from measured random vibration," Journal of Sound and Vibration, vol. 255, no. 3, pp. 549-554, 2002.

[3] K. S. Mohammad, K. Worden, and G. R. Tomlinson, "Direct parameter estimation for linear and nonlinear structures," Journal of Sound and Vibration, vol. 152, no. 3, pp. 471-499, 1992.

[4] J. V. Tomme, "Evaluation of damping measurements in materials and structures," in Proceedings of the 13th International Seminar on Modal Analysis, 1988. 
[5] B. J. Lazan, Damping of Materials and Members in Structural Mechanics, Oxford Pergamon Press, 1968.

[6] J. Naprstek, "Identification of linear and non-linear dynamic systems with parametric noises," in Proceedings of the 4th International Conference on Stochastic Structural Dynamics, 1999.

[7] p. J. Murtagh and B. Basu, "Identification of equivalent modal damping for a wind turbine at standstill using Fourier and wavelet analysis," Journal of Multi-body Dynamics, vol. 221, no. 4, pp. 1464-4193, 2007.

[8] T. S. Jang, H. S. Choi, and S. L. Han, "A new method for detecting non-linear damping and restoring forces in non-linear oscillation systems from transient data," International Journal of NonLinear Mechanics, vol. 44, no. 7, pp. 801-808, 2009.

[9] T. S. Jang, "Non-parametric simultaneous identification of both the nonlinear damping and restoring characteristics of nonlinear systems whose dampings depend on velocity alone," Mechanical Systems and Signal Processing, vol. 25, no. 4, pp. 1159-1173, 2011.

[10] T. S. Jang, H. S. Baek, H. S. Choi, and S. K. Lee, "A new method for measuring nonharmonic periodic excitation forces in nonlinear damped systems," Mechanical Systems and Signal Processing, vol. 25, no. 6, pp. 2219-2228, 2011.

[11] T. S. Jang, J.W. Son, S. L. Han et al., "A numerical investigation on nonparametric identification of nonlinear roll damping moment of a ship from transient response," The Open Ocean Engineering Journal, vol. 3, pp. 100-107, 2010.

[12] T. S. Jang, "A novel method for non-parametric identification of nonlinear restoring forces in nonlinear vibrations from response data: a dissipative nonlinear dynamical system," Ships and Offshore Structures. In press.

[13] T. S. Jang, S. H. Kwon, and J. H. Lee, "Recovering the functional form of the nonlinear roll damping of ships from a free-roll decay experiment: an inverse formulism," Ocean Engineering, vol. 37, no. 14-15, pp. 1337-1344, 2010.

[14] T. S. Jang, S. H. Kwon, and S. L. Han, "A novel method for non-parametric identification of nonlinear restoring forces in nonlinear vibrations from noisy response data: a conservative system," Journal of Mechanical Science and Technology, vol. 23, no. 11, pp. 2938-2947, 2010.

[15] J. A. Murdock, Perturbations, John Wiley \& Sons, New York, NY, USA, 1991.

[16] C. W. Groetsch, Inverse Problems in the Mathematical Sciences, Vieweg, Braunschweig, Germany, 1993.

[17] T. S. Jang, H. S. Baek, S. L. Hana, and T. Kinoshita, "Indirect measurement of the impulsive load to a nonlinear system from dynamic responses: inverse problem formulation," Mechanical Systems and Signal Processing, vol. 24, no. 6, pp. 1665-1681, 2010.

[18] T. S. Jang and T. Kinoshita, "An ill-posed inverse problem of a wing with locally given velocity data and its analysis," Journal of Marine Science and Technology, vol. 5, no. 1, pp. 16-20, 2000.

[19] T. S. Jang, H. S. Choi, and T. Kinoshita, "Numerical experiments on an ill-posed inverse problem for a given velocity around a hydrofoil by iterative and noniterative regularizations," Journal of Marine Science and Technology, vol. 5, no. 3, pp. 107-111, 2000.

[20] T. S. Jang, H. S. Choi, and T. Kinoshita, "Solution of an unstable inverse problem: wave source evaluation from observation of velocity distribution," Journal of Marine Science and Technology, vol. 5, no. 4, pp. 181-188, 2001.

[21] T. S. Jang, S. H. Kwon, and B. J. Kim, "Solution of an unstable axisymmetric Cauchy-Poisson problem of dispersive water waves for a spectrum with compact support," Ocean Engineering, vol. 34, no. 5-6, pp. 676-684, 2007.

[22] T. S. Jang and S. L. Han, "Application of tikhonov's regularization to an unstable two dimensional water waves: spectrum with compact support," Ships and Offshore Structures, vol. 3, pp. 41-47, 2008. 


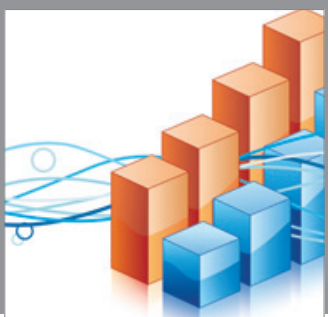

Advances in

Operations Research

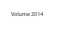

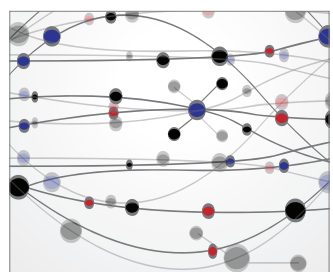

\section{The Scientific} World Journal
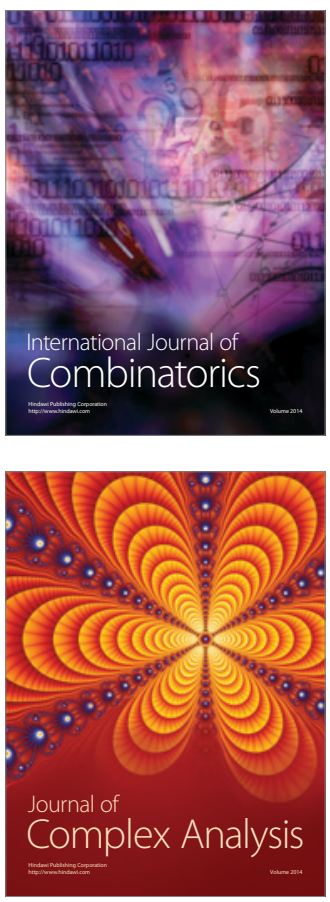

International Journal of

Mathematics and

Mathematical

Sciences
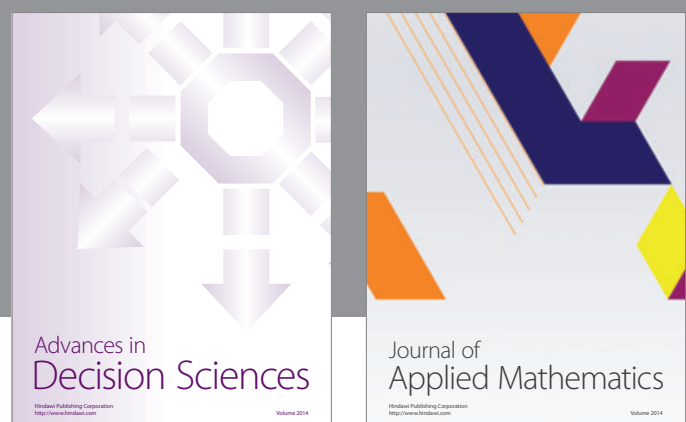

Journal of

Applied Mathematics
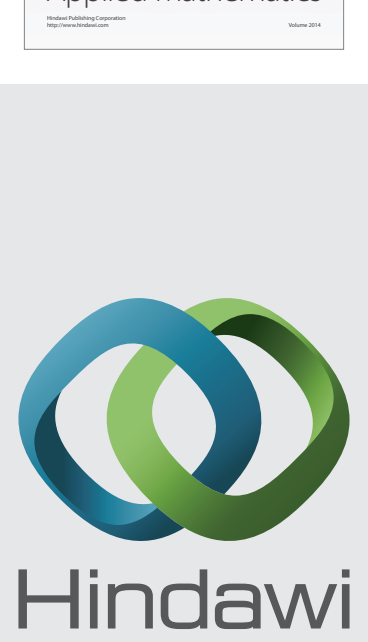

Submit your manuscripts at http://www.hindawi.com
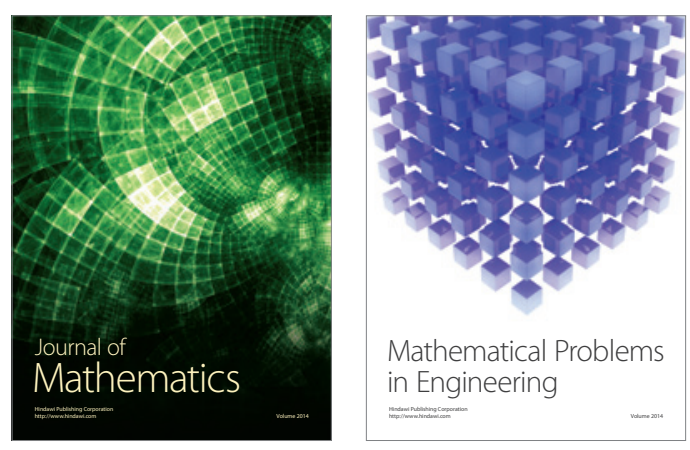

Mathematical Problems in Engineering
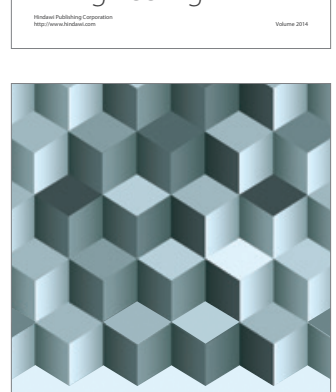

Journal of

Function Spaces
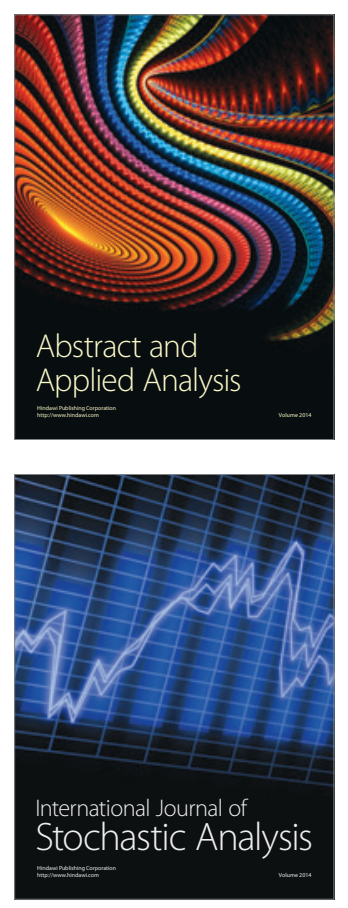

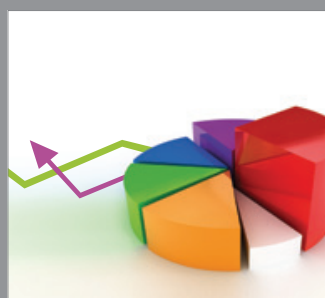

ournal of

Probability and Statistics

Promensencen
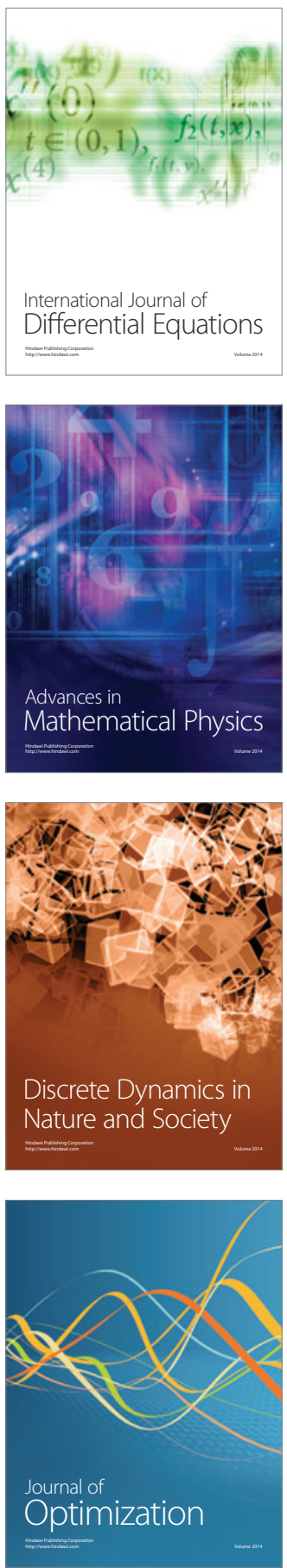